\title{
Listening Music in Reducing Anxiety for Regional Anesthesia- Cases
}

\author{
1 University Malaya ${ }^{1}$; mrahmat@ummc.edu.my \\ 2 University Malaya²; marzida@ummc.edu.my \\ * Correspondence: mrahmat@ummc.edu.my.
}

Mohd Rahmat Bin Abdul Hamid 1, Marzida Binti Mansor ${ }^{2}$ and Mohd Fitry Bin Zainal Abidin 2,*

\begin{abstract}
In this study, the effects of music therapy on anxiety for patients undergoing regional anaesthesia in an operating room was succinctly investigated. This investigation was largely based on the adapted Hospital Anxiety and Depression Score (HADS) and Spielberger State-Trait Anxiety Inventory (STAI-S), of patients undergoing regional anaesthesia in an operating room. A randomized control trial was performed on 90 patients due for surgery. The selected patients for regional anaesthesia were allocated to either the music therapy group who listened to music using headphones for the entire surgery or the no-treatment control group. Based on the findings, it has been conclusively demonstrated that music can decrease the patient's anxiety level. According to the socio-demographic evaluation, elderly patients have the highest stress hormones levels when compared to young patients. Although elderly patients are more likely to choose religious songs to help them relax, cortisol analysis revealed an increase in cortisol levels among the elderly compared with younger patients. As a result, music is especially important to be delivered to elderly patients. Nonetheless, there is no restriction against administering music to elderly patients because evidence from the Hospital Anxiety and Depression Score (HADS) and State-Trait Anxiety Inventory (STAI-S) has shown that music helps to shift their attention away from pain and complications and makes them feel tranquil. Similarly, the HADS and modified Spielberger STAI (STAI-S) analyses demonstrate a substantial outcome for both groups, with respondents responding positively. The study found that listening to music during regional anaesthesia might help people feel less worried.
\end{abstract}

Keywords: Anesthesia; Anxiety; Regional anaesthesia; Music Therapy

\section{Introduction}

An approximate 266-360 million surgical operations are performed globally yearly [1]. Preoperative anxiety and postoperative discomfort are common in patients undergoing surgery; current statistics estimated that $75 \%$ of patients before surgery feel apprehensive, despite anxiety-reduction treatments [2]. Preoperative anxiety can enhance postoperative pain, and notwithstanding measures to minimize postoperative pain, 40-65 per cent of patients report mild to severe distress following surgery [1]. Regional anaesthesia has grown in popularity in recent times. It is classified into two types: central neural-axial block and peripheral nerve block. Aside from avoiding the risks associated with general anaesthesia regional anaesthesia delivers superior analgesia intra-operatively and postoperatively, making it a more desirable anaesthetic alternative in many instances [3].

However, there is a significant prevalence of fear for regional anaesthesia and its procedures which can affect the patients' decision and postoperative anxiety [4]. Most anxious patients prefer to be asleep during the surgery. This can be achieved by giving either intermittent or continuous sedation throughout the surgery. The administration of sedatives has many advantages which usually makes the patient feels comfortable with an associated short-term memory loss which erases unpleasant memories. However, if these drugs are misused or abused, they can suppress the cardiovascular and respiratory systems, lower core temperature, or severely lower patients' blood pressure [5]. Moreover, some patients are not suitable for sedation, for instance, obstetric and elderly patients. In the case of peripheral nerve block, ideally, patients need to be awake to minimize the risk 
of damaging the particular nerve, hence the sedation is relatively contraindicated [6]. Taking into account the above challenges of pharmacological sedation; non-pharmacological anxiolytics might be a good alternative for the patients [7-9]. There are several methods of non-pharmacological anxiolytics such as music therapy, virtual reality video and aromatherapy [10-12]. Music therapy has been shown to have a sedative effect and reduce anxiety in patients undergoing regional anaesthesia [9]. Music therapy has also been suggested as a multimodal non-pharmacological intervention strategy to alleviate pain in a systematic review of music interventions in perioperative care [13-14]. Music can be a safe, cost-effective way to address patient needs and perhaps improve patient satisfaction, both of which are increasingly important in our present healthcare system [15]. In this study music therapy was used as an anxiolytics method during the regional anaesthesia for trauma orthopaedic patients. The primary outcome of this research is to reduce anxiety, assessed by Hospital Anxiety and Depression Score (HADS) and Spielberger State-Trait Anxiety Inventory (Spielberger STAI). The secondary outcomes were the hemodynamic variables such as the blood pressure systolic blood pressure (SBP), diastolic blood pressure (DBP), heart rate (HR), respiration rate (RR) and oxygen saturation level $\left(\mathrm{O}_{2}\right)$. This study aims to identify the relationship between the effects of listening to music with sociodemographic variables. Also, the effect of listening to music in reducing the level of anxiety using was succinctly investigated using Hospital Anxiety Depression Scoring (HADS) for regional anaesthesia cases at the orthopaedic operating theatre of the University Malaya Medical Centre, Malaysia.

\section{Materials and Methods}

\subsection{Determination Sample Size}

The G-power 3.010 software was used to calculate the sample size. Given the power $=0.8,=0.05$, effect size $=0.3$, and two-tailed test, a sample size of 90 patients was required. The decision to treat was based on the experimental group's $(n=45)$ and control group's $(n=45)$ double parallel arm equality. The samples in the experimental and control groups were randomised using the randomizer.org software.

\subsection{Ethical Consideration and Study Method}

This study was sponsored by the Malaysian Society of Anesthesiologists (MSA) with Universiti Malaya Ethics Committee Trial Registry Number of 201794-5541 (MRECUMMC). . The quantitative method is needed to determine positive and negative feedback from the patients after music therapy was being applied to them. The data of hemodynamic (Blood pressure, heart rate, and Oxygen saturation were taken. Patients listened to music using headsets through an MP3 player throughout the surgical operation to reduce external noise from the operating room and boost concentrate on music. The signal width was re-adjusted to reach a suitable volume to hear the whole dynamic range of the musical composition. The single-arm for the experimental group $(n=45)$ with music, hemodynamic data was taken for 3 sessions. In the first session, the patient was made to listen to music for 30 minutes inside the ward before sending them to operation theatre (baseline), while in the second session they were made to listen to the music 30 minutes after regional anaesthesia was given. Finally, in the third session, they were made to listen to the music 30 minutes after music therapy was off.

The trial design for the different arms consisted of two parts: (1) with music, which will get music intervention throughout the surgical process, and (2) a control group that will not get music intervention and will get routine care during surgery. Besides the requirements for intradural anaesthesia (without sedation), no extra analgesic or anxiolytic medicines were supplied to patients in both the MT and control groups. An investigator anonymous to the patient's group assessed the study variables in each patient randomly of the assigned group, before and after the experimental intervention. All data from the experimental and control group were analysed for double parallel arm analysis. Serum 
cortisol was taken for each group (Recovery Bay) to compare any significance of music therapy effect, as to if they were either an increase or decrease in the level of the serum cortisol for each group.

\subsection{Data Collection}

Respondents who met the inclusion criteria were given the questionnaire and permission. During the briefing time, the patients were instructed on the procedures before their voluntary agreement was sought. Before receiving regional anaesthetic at the UMMC Operation Theater, the responders signed a written permission form. There were 90 (45-intervention group and 45-control group) patients included in this study. The patients were divided into two main groups; consisting of music and no music group. Each responder was allocated a code number before the surgery to preserve their confidentially and for follow-up, purposes to gather data following regional anaesthesia to administer music therapy. Before transferring patients to the operating room, the hemodynamic data (blood pressure, heart rate, respiration rate, and oxygen saturation) are collected in the ward for 30 minutes. The randomizer.org programme was used to assign patients to groups. Each randomization result was placed inside an envelope with simple instructions determining whether the participants would get music therapy as an experimental group or no music therapy as a control group.

\section{Results}

\subsection{Analysis of Demographic Information of Respondents}

The demographic information of the respondents from this study revealed the gender, age, ethnic group, education level, marital status, type of song and duration (Table 1). The patients were divided into two main groups; consisting of music and no music group. The results obtained showed that there was no significant difference in the distribution of patients based on the groups with a p-value more than 0.05 except education level ( $p$-value=0.024). The analysis of socio-demographic data showed that none of the respondents rejected or withdrew from the study. It proved that all categories of ages, ethnics and marital status wants the music therapy to be applied to them during the operation. Descriptive analysis was used to find out which song had the highest demand for both groups; most of the respondents preferred religious songs. Total numbers of 19 respondents $(42.2 \%)$ for the interventional group and 17 respondents $(37.8 \%)$ for the control group choose the religious song. However, there is a difference that existed amidst the selected type of song between ballad and pop-rock for both groups. The intervention group had more preference for ballad song (14 respondent's equal to 31.1\%) in the second place compared to the control group (13 respondents equal to $28.9 \%$ ). However, the different selected type of pop song can be revealed from the control group whereby the respondents in this group had more preference for a pop song (15 respondents equal to $33.3 \%$ ) compared to the intervention group with the smallest percentage (12 respondents equal to $26.7 \%$ ). The result probably was because most of the respondents came from the married group with the highest percentage for the intervention group at 31.1 respondents $(68.9 \%)$ with the same percentage going to the control group. The single status of respondents showed the lowest percentage with only 14 respondents (31.1\%) for both groups. The ages of the respondents who preferred to choose the religious song during the operation to make them stay calm seem to be a vital factor for their choice. Based on the analysis, the ages of 41years -60 years which represents 16 respondents and equal to $35.6 \%$ and those more than 60 years represented 14 respondents which were equal to $31.1 \%$, summing up to a total of $66.7 \%$ compared to $33.3 \%$ for categories of ages of 21 years to 40 years and below 20 years. Finally, the religious song has been chosen as the top priority to be applied for regional anaesthesia cases at the operating theatre to reduce the anxiety level among the patients with ages between 41 years and above. However, ballad and pop song can be added on as choice to be applied for group of ages below 40 years. Somehow, 
any categorical of ages still can choose any preferable type of song to make them less nervous and more calmed during the period of operation under regional anaesthesia.

Table 1. Demographic Information of the Respondents.

\begin{tabular}{|c|c|c|c|}
\hline Variables & Music-Frequency (\%) & No Music-Frequency (\%) & p-value \\
\hline Gender & & & $>0.999$ \\
\hline Male & $28(62.2)$ & $29(64.4)$ & \\
\hline Female & $17(37.8)$ & $16(35.6)$ & \\
\hline Age & & & 0.265 \\
\hline$\leq 20$ years old & $0(0.0)$ & $2(4.4)$ & \\
\hline $21-40$ years old & $15(33.3)$ & $14(31.1)$ & \\
\hline $41-60$ years old & $16(35.6)$ & $10(22.2)$ & \\
\hline More than 60 years old & $14(31.1)$ & $19(42.2)$ & \\
\hline Ethnic group & & & 0.243 \\
\hline Malay & $26(57.8)$ & $20(44.4)$ & \\
\hline Chinese & $11(24.4)$ & $17(37.8)$ & \\
\hline Indian & $6(13.3)$ & $8(17.8)$ & \\
\hline Others & $2(4.4)$ & $0(0.0)$ & \\
\hline Education level & & & 0.024 \\
\hline Primary & $15(33.3)$ & $13(28.9)$ & \\
\hline Secondary & $16(35.6)$ & $14(31.1)$ & \\
\hline Diploma & $12(26.7)$ & $6(13.3)$ & \\
\hline Degree & $2(4.4)$ & $12(26.7)$ & \\
\hline Marital status & & & $>0.999$ \\
\hline Single & $14(31.1)$ & $14(31.1)$ & \\
\hline Married & $31(68.9)$ & $31(68.9)$ & \\
\hline Types of song & & & 0.786 \\
\hline Pop & $12(26.7)$ & $15(33.3)$ & \\
\hline Balada & $14(31.1)$ & $13(28.9)$ & \\
\hline Religion & $19(42.2)$ & $17(37.8)$ & \\
\hline Duration (minutes) & & & 0.282 \\
\hline
\end{tabular}

3.2. Descriptive Analysis for Hospital Anxiety and Depression Score (HADS)

From Table 2, the mean score of the No music group (M=15.58, SD: 2.231) is higher than that of the Music group $(M=11.91, S D=3.218)$. This can however indicate that with regards to the Hospital Anxiety and Depression Score analysis carried out on both groups; those in the No Music group had higher scores. Hence, it can be inferred that when there is no music applied to patients during operation, their anxiety and depression level increases, but on the other hand, if music intervention is being applied; it helps in reducing their anxiety and depression levels. 
Table 2. Result descriptive statistic through descriptive analysis for HADS.

\begin{tabular}{ccccc}
\hline & Minimum & Maximum & Mean & Std. Deviation \\
\hline Music & 5 & 18 & 11.91 & 3.218 \\
No music & 7 & 18 & 15.58 & 2.231 \\
\hline
\end{tabular}

From the HADS analysis shown that the Music Group Table 3 based on the frequency test, those patients who indicated that they needed music to reduce their depression and anxiety levels were around 30 out of the total 45 participants. This hence reveals the importance of the music intervention for patients and how it can help in reducing their hospital anxiety and depression levels. From the No Music group (Table 3), those who needed music during the operation level were even more than those who indicated in the Music group as discussed earlier. Out of the 45 participants in this group, 44 of them indicated that they needed Music intervention to help reduce their anxiety and depression levels, this means that these patients might have passed through series of anxiety and depression as a result of the non-appliance of Music therapy during the operating period. Hence, Music is a very important means of reducing anxiety and depression among operating patients [16].

Table 3. Result in descriptive statistic through frequency analysis of HADS for Music Group (interventional group) and N0 Music Group (control group) after recoding the variable base on the anxiety level.

\begin{tabular}{|c|c|c|c|c|c|}
\hline \multicolumn{6}{|c|}{ HADS-_Music Group } \\
\hline & & Frequency & Per cent & Valid & Cumulative \\
\hline & & & & Percent & Percent \\
\hline \multirow[t]{4}{*}{$\mathrm{N}(45)$} & NORMAL(1) & 3 & 6.7 & 6.7 & 6.7 \\
\hline & BORDERLINE(2) & 12 & 26.7 & 26.7 & 33.3 \\
\hline & NEED MUSIC(3) & 30 & 66.7 & 66.7 & 100.0 \\
\hline & Total & 45 & 100.0 & 100.0 & \\
\hline \multicolumn{6}{|c|}{ HADS-No Music Group } \\
\hline & & Frequency & Per cent & Valid Percent & Cumulative \\
\hline & & & & & Percent \\
\hline \multirow[t]{3}{*}{$\mathrm{N}(45)$} & NORMAL(1) & 1 & 2.2 & 2.2 & 2.2 \\
\hline & NEED MUSIC(3) & 44 & 97.8 & 97.8 & 100.0 \\
\hline & Total & 45 & 100.0 & 100.0 & \\
\hline
\end{tabular}

${ }^{*}$ Recode score to (1) normal, (2) borderline and (3) need music therapy

From Table 4, which combines both groups, it can be seen that those in the No Music group had a higher score of depression and anxiety, which again shows the relevance of applying music intervention during operation. Also, the data was analysed using the Independent t-Test. There was a significant difference in C2, C3, C5, C6 and total C score between music and no music group with a $\mathrm{P}$ value less than 0.05 . However, the result of the Chi-Square test for all variables (C1-C7) revealed that there was no significant difference in HADS between music and no music group with a p-value greater than 0.05 . 
Table 4. Result in descriptive statistic through descriptive and correlation analysis of HADS for Music Group vs N0 Music Group after recoding the variable base on the anxiety level.

\begin{tabular}{|c|c|c|c|c|c|c|}
\hline \multicolumn{7}{|c|}{ Descriptive Statistics } \\
\hline & $\mathrm{N}$ & Minimum & Maximum & Mean & & Std. Deviation \\
\hline HADS-music & 45 & 1 & 3 & 2.60 & & .618 \\
\hline HADS-no music & 45 & 1 & 3 & 2.96 & & .298 \\
\hline \multicolumn{7}{|c|}{ Recode score to (1) normal, (2) borderline and (3) need music therapy. } \\
\hline \multicolumn{7}{|c|}{ Correlation analysis } \\
\hline & Mean & \multicolumn{2}{|c|}{ Std. Deviation } & \multicolumn{3}{|c|}{$\mathrm{N}$} \\
\hline music & 11.91 & \multicolumn{2}{|c|}{3.218} & \multicolumn{3}{|c|}{45} \\
\hline no music & 15.58 & \multicolumn{2}{|c|}{2.231} & \multicolumn{3}{|c|}{45} \\
\hline \multicolumn{7}{|c|}{ (Score of 0-7 considered normal, 8-10 borderlines and above 11 indicated music therapy). } \\
\hline Variables & \multicolumn{3}{|c|}{ the p-value for Independent $t$-test } & the $p$-value & \multicolumn{2}{|c|}{ for the Chi-Square test } \\
\hline $\mathrm{C} 1$ & \multicolumn{3}{|c|}{0.346} & \multicolumn{3}{|l|}{0.340} \\
\hline $\mathrm{C} 2$ & \multicolumn{3}{|c|}{$<0.001$} & \multicolumn{3}{|l|}{0.720} \\
\hline C3 & \multicolumn{3}{|c|}{$<0.001$} & \multicolumn{3}{|l|}{0.210} \\
\hline $\mathrm{C} 4$ & \multicolumn{3}{|c|}{0.588} & \multicolumn{3}{|l|}{0.173} \\
\hline C5 & \multicolumn{3}{|c|}{$<0.001$} & \multicolumn{3}{|l|}{0.161} \\
\hline C6 & \multicolumn{3}{|c|}{$<0.001$} & \multicolumn{3}{|l|}{0.160} \\
\hline C7 & \multicolumn{3}{|c|}{0.057} & \multicolumn{3}{|l|}{0.227} \\
\hline Total score & \multicolumn{3}{|c|}{$<0.001$} & & & \\
\hline
\end{tabular}

\subsection{Descriptive analysis for State Trait Anxiety Inventory (STAI)}

From this Table 6 above, we can see that the State-Trait Anxiety Inventory for the patients in the No Music group has a greater score than their counterpart in the Music group. The Mean for the STAI_NO MUSIC group is 54.04 (SD = 2.899), which is greater than that of those in the music group. This can hence indicate that the patients in the NO Music group tend to have a higher anxiety level than those in the music group. Therefore, Music intervention can be a very great way of reducing anxiety in patients undergoing the operation. Data were analysed using the Chi-Square test to determine the association between STAI outcome and the groups (music and no music). Based on the test, there was a significant association between the groups with all STAI $(p<0.05)$ except the anguished expression. The results obtained from each patients distribution based on the studied groups show a significant association among the variables such as set 1: calmed down, set 2: safe, set 3: tense, set 4: annoyed, set 5: comfortable, set 4: upset, set 5: concerned with future misfortunes, set 6: relaxed, set 8: at ease, set 9: self-confidence, set 10: nervous, set 11: restless, set 12: downhearted, set 13: rested, set 14: satisfied, set 15: concerned, set 16: stunned, set 17: happy, and set 18: I feel good. The only exception to this is set 7: anguished; which has an insignificant contribution on the groups and STAI outcome. 
Table 6. Analysis result of descriptive statistic base on frequency analysis for both groups.

\begin{tabular}{ccc}
\hline & Statistics \\
\hline N & STAI_MUSIC & STAI_NO MUSIC \\
Mean & 45 & 45 \\
Median & 48.87 & 54.04 \\
Mode & 51.00 & 54.00 \\
Std. Deviation & 52 & 57 \\
\multicolumn{1}{c}{ Score } & 4.595 & 2.899 \\
\hline & $20-39$ & \multicolumn{2}{c}{ remark } \\
& $40-59$ & Low anxiety (1) \\
& $60-79$ & Mild anxiety(2) \\
& $>80$ & High level of anxiety(3) \\
\end{tabular}

\subsection{Correlation of Cortisol and Age}

From the correlation regression table 4.54, the analysis derived that there are some relationship between age and cortisol with a $p<0.05$, hence $p=0.002$. However, for the case of sex and cortisol, there is no significant relationship from the correlation regression test, $p>0.05$, hence $p=0.257$. But moving further into the Regression test proper to find out more in-depth facts for the relationships between the dependent variable cortisol and the independent variables (age and sex), we can see that age is a positive predictor with a B value of $4.011, t=2.794$ and $p=0.006$ which shows its significance and strong relationship with Cortisol. On the other hand, sex has proven not to be a good predictor for Cortisol, it has a negative $B$ value of -6.788 (this shows it's a negative predictor), $t=-0.124$ and $\mathrm{p}=0.902$ which shows its non-significance and its weakness in prediction.

Table 7. The residual statistic for the dependent variable (cortisol).

\begin{tabular}{|c|c|c|c|c|c|}
\hline \multicolumn{6}{|c|}{ Residuals Statistics } \\
\hline & Minimum & Maximum & Mean & Std. Deviation & $\mathrm{N}$ \\
\hline Predicted Value & 306.54 & 598.14 & 443.93 & 73.523 & 90 \\
\hline Residual & -366.012 & 839.804 & .000 & 238.466 & 90 \\
\hline Std. Predicted Value & -1.869 & 2.097 & .000 & 1.000 & 90 \\
\hline Std. Residual & -1.518 & 3.482 & .000 & .989 & 90 \\
\hline
\end{tabular}

Hence, from this analysis, we can infer that age is a good predictor of Cortisol, that is to say, the rate of the increase in the blood of patients can depend on their age [17]. However, their sex or gender might not have any association with regards to the increase in the blood of the patients. Also, from the Model Summary, we can see that the R square value is $87 \%$ which is relatively high and shows that the model is very good and well fit. To further buttress the validity of the above test, a scatter plot for the relationship between age and Cortisol is being presented in Figure 1. 


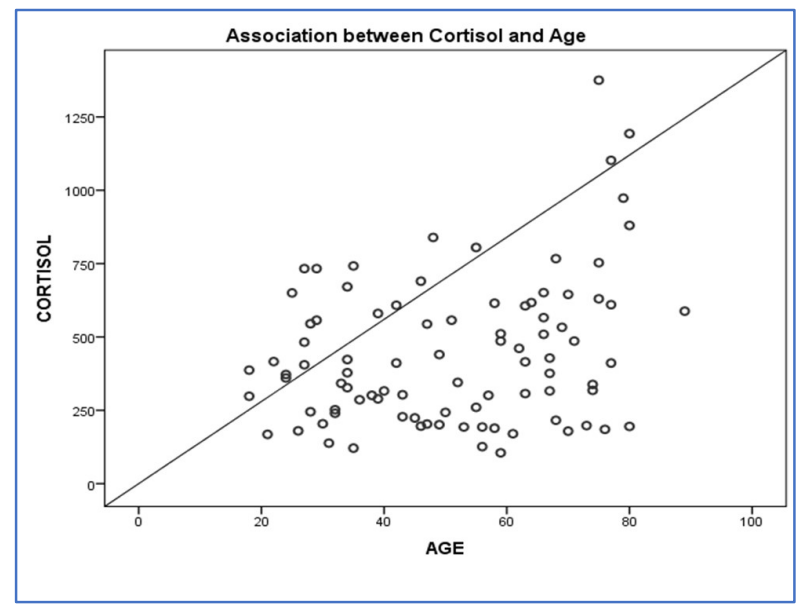

Figure 1. Scatter plot of the association between cortisol and age.

From the scatter plot above, it is evident that the dots are well scattered and sloppily spread around each other. This shows that a strong relationship exists between age and Cortisol. Another interesting point we can infer from this scatter plot is that as the age of the patient increases, the cortisol (that is the increase in the blood) also rises. It means older patients are likely to be having more cases of anxiety during operation cases than younger patients. Therefore, the Music intervention could be of much importance to the older patients during operation.

\section{Conclusions}

Music therapy, a non-pharmacologic remedy, is an effective treatment that has no adverse effects and has a beneficial effect on patients who are under local anaesthetic. It can also help with anxiety, discomfort, and vital signs. The results of this study provide evidence that pain and anxiety are reduced while listening to music. Based on the findings from the Adapted HADS and Spielberger STAI, it was known that the patients enjoyed the music, reporting that the music helped them to forget about their pain and thoughts for a while during operation after receiving regional anaesthesia and improved their general mood as well as their hemodynamic variables. The use of this intervention could be implemented into the routine plan of care for regional anaesthesia cases at the operation theatre. The intervention poses no risks and has the potential to limit the number of opioids necessary to achieve pain relief, which decreases the chances of experiencing the side effects of opioids, such as respiratory depression.

Conflicts of Interest: The authors declare no conflict of interest.

\section{References}

1. Kühlmann, A. Y. R., De Rooij, A., Kroese, L. F., Van Dijk, M., Hunink, M. G. M., \& Jeekel, J. (2018). Meta-analysis evaluating music interventions for anxiety and pain in surgery. The British journal of surgery, 2018, 105(7), 773.

2. De Oliveira GS Jr, Holl JL, McCarthy RJ, Butt ZA, Nouriel J, McCaffery K. Overestimation of mortality risk and preoperative anxiety in patients undergoing elective general surgery procedures: a propensity matched analysis. International Journal of Surgery, 2014; 12: 1473-1477.

3. Kakar, E., Billar, R. J., Van Rosmalen, J., Klimek, M., Takkenberg, J. J., \& Jeekel, J. Music intervention to relieve anxiety and pain in adults undergoing cardiac surgery: a systematic review and meta-analysis. Open heart, 2021, 8(1), e001474.

4. Chandrababu, R., Ramesh, J., Sanatombi Devi, E., Nayak, B. S., \& George, A. Effectiveness of music on anxiety and pain among cardiac surgery patients: A quantitative systematic review and meta-analysis of randomized controlled trials. International Journal of Nursing Practice, 2021, e12928.

5. Orman, O., Yeniocak, T., Baydar, M., Şencan, A., İpteç, M., \& Öztürk, K. The effect of wide-awake anesthesia, intravenous regional anesthesia, and infraclavicular brachial plexus block on cost and clinical scores of patients undergoing hand surgery. Hand Surgery and Rehabilitation. 2021. 
6. Jasper, A. O., Onohwakpor, E. A., \& Akhator, A. High-risk surgeries, anesthetic challenges, and real benefits of peripheral regional techniques revisited. Nigerian. Journal of Clinical Practice, 2021, 24(5), 782.

7. Song, M., Li, N., Zhang, X., Shang, Y., Yan, L., Chu, J., Xu, Y. Music for reducing the anxiety and pain of patients undergoing a biopsy: A meta-analysis. Journal of advanced nursing, 2018, 74(5), 1016-1029.

8. Stern, C. Music interventions for preoperative anxiety. International journal of evidence-based healthcare, 2013, 11(3), $208-209$.

9. Tola, Y. O., Chow, K. M., \& Liang, W. Effects of non-pharmacological interventions on preoperative anxiety and postoperative pain in patients undergoing breast cancer surgery: A systematic review. Journal of Clinical Nursing, 2021

10. Eroglu, A. Regional anesthesia techniques in orthopedic surgery. J Anaesth Crit Care Open Access, 2016, 5(2), 00182.

11. Lobo, D. Non Pharmacological Interventions to Manage Cancer-Related Fatigue (CRF)-An Overview. Journal of Complementary and Alternative Medical Research, 2021, 42-51.

12. Scrine, E. (2021). The limits of resilience and the need for resistance: Articulating the role of music therapy with young people within a shifting trauma paradigm. Frontiers in Psychology, 2021, 12, 26.

13. Missig, G., Mei, L., Vizzard, M. A., Braas, K. M., Waschek, J. A., Ressler, K. J., May, V. Parabrachial PACAP activation of amygdala endosomal ERK signaling regulates the emotional component of pain. Biological psychiatry, 2017, 81(8), 671.

14. Graff, V., Cai, L., Badiola, I., \& Elkassabany, N. M. Music versus midazolam during preoperative nerve block placements: a prospective randomized controlled study. Regional Anesthesia \& Pain Medicine, 2019, 44(8), 796-799.

15. Fan, M., \& Chen, Z. A systematic review of non-pharmacological interventions used for pain relief after orthopedic surgical procedures. Experimental and Therapeutic Medicine, 2020, 20(5), 1-1.

16. Kahna, M., \& Belgat, W. G. The Contribution of Music Therapy to the Operating Room: A Randomized Control Study. MAR Case Reports; Medical and Research Publications: Stoke-on-Trent, UK. 2020.

17. Takahashi, T., \& Matsushita, H. Long-term effects of music therapy on elderly with moderate/severe dementia. Journal of Music Therapy, 2006, 43(4), 317-333. 